DFTT $15 / 04$

July 2004

\title{
Scaling of Power Corrections for Angularities from Dressed Gluon Exponentiation
}

\author{
Carola F. Berger ${ }^{a 1}$ and Lorenzo Magnea ${ }^{b 2}$ \\ ${ }^{a}$ I.N.F.N., Sezione di Torino \\ Via P.Giuria 1, I-10125 Torino, Italy \\ ${ }^{b}$ Dipartimento di Fisica Teorica, Università di Torino \\ and I.N.F.N., Sezione di Torino \\ Via P.Giuria 1, I-10125 Torino, Italy
}

\begin{abstract}
We study power corrections to a recently introduced family of event shapes, the class of angularities, within the formalism of dressed gluon exponentiation (DGE). We find that the universal scaling rule for the leading power corrections deduced from resummation also holds when taking renormalon enhancements into account. The scaling is due to boost invariance of eikonal dynamics in the twojet limit, which we recover in the context of DGE. Furthermore, dressed gluon exponentiation provides an ansatz for non-leading power corrections that violate the scaling. These non-leading corrections are further suppressed by non-integer powers of the hard scale.
\end{abstract}

\footnotetext{
${ }^{1}$ e-mail: carola.berger@to.infn.it

${ }^{2}$ e-mail: magnea@to.infn.it
} 


\section{Introduction}

Event shapes [1, 2, 3] are infrared-safe generalizations of jet cross sections, and they are among the best suited observables to test our understanding of QCD. Event shapes distributions, in particular, describe the behavior of colored radiation in the final state of hard collisions in all of phase space. Thus, although infrared safe, they are sensitive to emission at all scales, and provide a unique tool to probe the interface between perturbative and non-perturbative QCD.

A consequence of the sensitivity of event shapes to long-distance effects is that perturbative calculations are far from straightforward even at high energies, where the strong coupling is sufficiently small. Specifically, in the narrow-jet limit radiation is dominated by secondary partons which are either soft or collinear to the primary quarks emitted at the hard scattering. As a consequence, fixed order computations receive large logarithmic corrections, which need to be resummed to all orders to obtain reliable quantitative predictions [4, 15, 6, 7, 8].

In addition to these large perturbative corrections, there are corrections due to confinement that cannot be treated within the perturbative expansion, and are suppressed by powers of the hard scale. Their main effect is to widen the distribution of radiation in the final state, shifting the peak away from the narrow jet limit. Although long-distance effects are intrinsically nonperturbative, the study of resummed perturbation theory can give valuable information about the size of their contributions to the cross section, as we will illustrate below.

In the following we will consider event shapes in $e^{+} e^{-}$annihilation. Power corrections to these observables have been the focus of intense research during the past several years (for recent reviews see for example [8, 9]). It turns out that for mean values of such event shapes the effects of power corrections can be efficiently summarized in terms of a single parameter, essentially a shift of the perturbative distribution by an amount proportional to an integer power of $1 / Q$, where $Q$ is the overall center-of-mass energy [10, 11, 12, 13. It was found that these additive corrections are to a certain degree universal. This universality property can be used phenomenologically for precise measurements of the strong coupling from event shapes [14].

The case of differential distributions, however, is more complicated. Distributions probe scales that are even smaller than those which dominate mean values, thus subleading power corrections also need to be taken into account. For a large class of such corrections this can be done by introducing a nonperturbative shape function [15]. Resummed perturbation theory, which displays sensitivity to soft emission at power accuracy, imposes constraints on models for these shape functions. Phenomenologically, the models are quite successful in describing the data, again with only a small set of nonperturbative parameters. Universality, however, is generically lost at the level of subleading power corrections, although certain classes of event shapes turn out to have 
closely related nonperturbative behavior [16, 17, 18, 19].

A class of event shapes of this type depending on a real parameter $a$ was introduced in 20]. These observables provide an interpolation between the thrust [1], corresponding to $a=0$, and the jet broadening [3, $a=1$. In this paper, we will refer to event shapes in this class as angularities. As shown in [20, 21, 22, 23, these event shapes can be studied analytically, as functions of $a$, both at the perturbative level, performing an all-order resummation of Sudakov logarithms, and at the level of long-distance effects. Using factorization arguments, it was found that leading power corrections to all the observables in the class of angularities are described by the same shape function, up to an overall scaling factor 23]. This remarkable property is closely related to the boost invariance of the soft radiation emitted in the two-jet limit.

We will study power corrections to the distributions of angularities by using renormalon methods. Specifically, we will use Dressed Gluon Exponentiation (DGE) 24 to build a model of the shape function. We will recover the scaling property discovered in [23], and we will study the pattern of violations of scaling due to the contributions of collinear radiation.

Dressed gluon exponentiation combines renormalon calculus with Sudakov resummation. On the one hand, taking into account single (dressed) gluon emission in the large $\beta_{0}$-limit, it identifies the leading source of factorially divergent behavior, characteristic of the asymptotic nature of the perturbative expansion. This is then upgraded to multi-gluon emission in the Sudakov limit by exponentiation of the single gluon result, employing as usual a Laplace transform to enforce momentum conservation. In the conventional resummed expressions, the asymptotic behavior manifests itself as a singularity in the perturbative running coupling at small scales, which introduces an ambiguity in the form of power corrections. The DGE formalism provides a definite prescription to deal with these ambiguities, and thus strongly constrains the form of nonperturbative shape functions. Dressed gluon exponentiation has been previously applied to several other event shapes, including the thrust and the C-parameter [17, 18, 19, 25], as well as to processes involving heavy quarks [26, and inclusive hadronic cross sections such as DIS and Drell-Yan [25, 27. Here we will apply it to study power corrections to angularities.

An interesting aspect of the study of angularities with this method is the fact that the scaling rule found in Ref. 23] was shown to be closely related to the boost invariance properties of the eikonal cross section describing soft emission in the two-jet limit. This property is not at all apparent in DGE, where in practice the single gluon cross section is computed with a "massive" gluon, along the lines of the dispersive approach. Since the introduction of the gluon virtuality breaks boost invariance, and since in addition is necessary to account for subleading logarithms in the large $n_{f}$ limit, it is not a priori clear whether the scaling will survive. Here we will show how the effects of boost invariance are recovered in the Sudakov region. In the two-jet limit, only the logarithmically divergent bremsstrahlung spectrum contributes to the dressed gluon cross section, and the gluon 
mass acts as an effective cutoff, with precisely the weight required to reconstruct an unweighted rapidity integral.

We start by briefly reviewing the definition of the class of angularities, and the scaling rule for nonperturbative corrections found by analyzing the resummed expression. In Secs. 3 and 4 we will then construct a model of the shape function for angularities by means of DGE. Concentrating on soft radiation, we will recover the scaling of leading power corrections, and study how it arises in the context of a massive gluon calculation. Finally, we will observe that DGE suggests an intricate, non-universal pattern of subleading power corrections arising from collinear radiation. All such corrections are however suppressed by non-integer powers of the hard scale, with a degree of suppression growing as the parameter $a$ becomes large and negative, where the event shape becomes correspondingly more inclusive. Technical details are given in the Appendix.

Although detailed phenomenological studies will have to be deferred to future work, when and if experimental data become available, we emphasize that such studies would be of considerable interest, and should be quite easy to perform for experimental collaborations. The scaling rule found in [23] and recovered here is in fact a clean and significant test of the behavior of soft radiation in QCD, and of the theoretical models employed in recent years to study power suppressed effects.

\section{The class of angularities}

We consider an $e^{+} e^{-}$annihilation event with center-of-mass energy $Q$, generating a final state $N$, which we will take for now to consist of massless particles. The angularity, with weight $a$, of the state $N$, is defined as [20]

$$
\tau_{a}(N)=\frac{1}{Q} \sum_{i \in N} p_{i \perp} e^{-\left|\eta_{i}\right|(1-a)}=\frac{1}{Q} \sum_{i \in N} \omega_{i}\left(\sin \theta_{i}\right)^{a}\left(1-\left|\cos \theta_{i}\right|\right)^{1-a},
$$

where $p_{i \perp}$ is the transverse momentum of particle $i$ relative to the thrust axis, $\eta_{i}$ is the corresponding pseudorapidity, $\eta_{i}=\ln \cot \left(\theta_{i} / 2\right)$, with $\theta_{i}$ the angle with respect to the thrust axis, and $\omega_{i}$ is the energy of particle $i$. The two definitions in Eq. (1I) are equivalent for massless particles.

The angularity distribution is defined as usual by summing over all final states, each weighed by its probability, according to

$$
\frac{d \sigma}{d \tau_{a}}=\frac{1}{2 Q^{2}} \sum_{N}|M(N)|^{2} \delta\left(\tau_{a}-\tau_{a}(N)\right),
$$

where $M(N)$ is the amplitude for the production of final state $N$.

The thrust axis can be defined as the axis with respect to which Eq. (11) is minimized at $a=0$. The parameter $a$ is adjustable in the range $-\infty<a<2$, with the upper limit 
set by infrared safety. Angularity with $a=0$ is essentially $1-T$, with $T$ the thrust [1], while angularity with $a=1$ corresponds to the jet broadening [3]. As discussed in [20], for $a \geq 1$ recoil effects become important, so that the resummation of Sudakov logarithms must be modified, and consequently the pattern of power corrections changes, as pointed out for the broadening $(a=1)$ in [7]. In this paper we will be mostly concerned with the case $a \leq 0$, where power corrections are under good control.

In the two-jet limit, $\tau_{a} \rightarrow 0$, the distribution in Eq. (2) has large perturbative corrections containing powers of $\ln \left(\tau_{a}\right)$, which have been resummed to all logarithmic orders, at leading power and for $a<1$, in [20]. As usual, the resummation is performed at the level of the Laplace transform of the distribution,

$$
\tilde{\sigma}(\nu, a)=\int_{0}^{\infty} d \tau_{a} \mathrm{e}^{-\nu \tau_{a}} \frac{d \sigma}{d \tau_{a}}
$$

At next-to-leading logarithmic (NLL) level, the resummation displays a nontrivial dependence on $a$,

$$
\begin{aligned}
\frac{1}{\sigma_{\text {tot }}} \tilde{\sigma}(\nu, a)=\exp \left\{2 \int_{0}^{1} \frac{d u}{u}\right. & {\left[\int_{u^{2} Q^{2}}^{u Q^{2}} \frac{d p_{\perp}^{2}}{p_{\perp}^{2}} A\left(\alpha_{s}\left(p_{\perp}\right)\right)\left(\mathrm{e}^{-u^{1-a} \nu\left(p_{\perp} / Q\right)^{a}}-1\right)\right.} \\
& \left.\left.+\frac{1}{2} B\left(\alpha_{s}(\sqrt{u} Q)\right)\left(\mathrm{e}^{-u(\nu / 2)^{2 /(2-a)}}-1\right)\right]\right\},
\end{aligned}
$$

where $A\left(\alpha_{s}\right)$ and $B\left(\alpha_{s}\right)$ are the well-known anomalous dimensions acting as kernels of Sudakov exponentiation. The intricate $a$ dependence of Eq. (4) simplifies at the level of leading logarithms, where one can easily invert the Laplace transform to find

$$
\frac{1}{\sigma_{\text {tot }}} \frac{d \sigma}{d \tau_{a}}=-\frac{2}{1-a / 2} \frac{\alpha_{s}}{\pi} C_{F} \frac{\ln \left(\tau_{a}\right)}{\tau_{a}} \exp \left[-\frac{1}{1-a / 2} \frac{\alpha_{s}}{\pi} C_{F} \ln ^{2}\left(\tau_{a}\right)\right]
$$

which displays a simple scaling with $1-a / 2$. This scaling of the perturbatively resummed cross section is however only approximate, and breaks down at NLL level, as can be seen in the explicit expressions given in the Appendix of Ref. [23].

Remarkably, the approximate scaling of the perturbative contribution with $1-a / 2$ is replaced at the level of leading power corrections by an exact scaling with $1-a$. To see this, one notes that the perturbative expression for the cross section given in Eq. (41) is ambiguous, due to the fact that the scale of the running coupling can vanish. As a consequence, at values of $\tau_{a} \sim \Lambda_{\mathrm{QCD}} / Q$ nonperturbative corrections must become dominant, and the perturbative expression needs to be supplemented by nonperturbative input to give a well-defined result. The structure of this nonperturbative correction can be deduced, following [15, 23], by introducing an infrared factorization scale $\kappa$ to 
cut off the transverse momentum integration in Eq. (44). The leading nonperturbative contribution arising from small transverse momenta can be evaluated by performing the integral over the Laplace variable $u$, keeping only terms scaling as powers of $\nu / Q$, while discarding terms suppressed by higher powers of the hard scale. The result can finally be written as a convolution of a perturbative contribution and a nonperturbative shape function, which in moment space is just a product,

$$
\begin{aligned}
\tilde{\sigma}(\nu, a) & =\tilde{\sigma}_{\mathrm{PT}}(\nu, \kappa, a) \tilde{f}_{a, \mathrm{NP}}\left(\frac{\nu}{Q}, \kappa\right)\left[1+\mathcal{O}\left(\frac{\nu}{Q^{2-a}}, \frac{\nu^{\frac{2}{2-a}}}{Q^{2}}\right)\right], \\
\ln \left[\tilde{f}_{a, \mathrm{NP}}\left(\frac{\nu}{Q}, \kappa\right)\right] & \equiv \frac{1}{1-a} \sum_{n=1}^{\infty} \lambda_{n}(\kappa)\left(-\frac{\nu}{Q}\right)^{n} .
\end{aligned}
$$

The shape function $\tilde{f}_{a, \mathrm{NP}}$ sums all power corrections of the form $(\nu / Q)^{n}$, with unknown nonperturbative coefficients $\lambda_{n}(\kappa)$, which can be formally expressed in terms of infrared moments of the cusp anomalous dimension $A\left(\alpha_{s}\right)$ as

$$
\lambda_{n}(\kappa)=\frac{2}{n n !} \int_{0}^{\kappa^{2}} \frac{d p_{\perp}^{2}}{p_{\perp}^{2}}\left(p_{\perp}^{2}\right)^{n / 2} A\left(\alpha_{s}\left(p_{\perp}^{2}\right)\right) .
$$

As explicitly indicated in Eq. (6), terms of order $\nu / Q^{2-a}$ and of order $\nu^{\frac{2}{2-a}} / Q^{2}$ have been neglected. At this level of accuracy, one finds that the only $a$-dependence of the shape function is through an overall factor $1 /(1-a)$, which leads to the scaling rule [21, 22, 23]

$$
\tilde{f}_{a, \mathrm{NP}}\left(\frac{\nu}{Q}, \kappa\right)=\left[\tilde{f}_{0, \mathrm{NP}}\left(\frac{\nu}{Q}, \kappa\right)\right]^{\frac{1}{1-a}} .
$$

The derivation of the scaling rule in Eq. (9) relies on two main assumptions. First, contributions from correlations between hemispheres are neglected, because the starting point is the NLL resummed cross section, which describes logarithmic corrections due to independent radiation off two back-to-back jets. In the more general resummed formula valid to all logarithmic orders [20], such correlations are present, but they contribute only starting at NNLL order. In addition, numerical studies indicate that inter-hemisphere correlations do not play an important role [16, 18. One can furthermore argue that correlations between hemispheres due to particles whose decay products enter both hemispheres become non-negligible in the same range of the parameter $a$ where also recoil effects need to be taken into account. The neglect of inter-hemisphere correlations is thus consistent with the resummation. The second assumption entering the derivation of Eq. (9) is that nonperturbative soft radiation has the same properties under boosts as the relatively harder perturbative component. A success of experimental tests of the scaling rule would thus show that boost invariant dynamics dominates the differential 
distributions at all scales and that coherent interjet radiation is nondominant in the relevant range of the parameter $a$.

Of course, even if the above assumptions hold, there are further corrections present, suppressed relative to the dominant ones, as indicated in Eq. (6). These corrections, which for thrust behave like $\nu / Q^{2}$, become important only in the extreme nonperturbative region $1-T \sim\left(\Lambda_{\mathrm{QCD}} / Q\right)^{2}$, and are unlikely to play a role for phenomenology. From a theoretical point of view, it may however be of some interest to compare the predictions of different models also for these subleading corrections. Considering the resummation, for example, one can keep terms neglected in Eq. (91), and parameterize them in terms of different integrals of the anomalous dimensions $A\left(\alpha_{s}\right)$ and $B\left(\alpha_{s}\right)$ in the infrared region. One finds that the pattern of subleading corrections can be characterized in terms of a subleading shape function as follows,

$$
\tilde{\sigma}(\nu, a)=\tilde{\sigma}_{\mathrm{PT}}(\nu, \kappa, a) \tilde{f}_{a, \mathrm{NP}}\left(\frac{\nu}{Q}, \kappa\right) \tilde{g}_{a, \mathrm{NP}}\left(\frac{\nu}{Q^{2-a}}, \kappa\right),
$$

where

$$
\begin{aligned}
\ln \left[\tilde{g}_{a, \mathrm{NP}}\left(\frac{\nu}{Q^{2-a}}, \kappa\right)\right] & \equiv \frac{1}{1-a} \sum_{n=1}^{\infty} \bar{\lambda}_{n}^{A}(\kappa, a)\left(-\frac{\nu}{Q^{2-a}}\right)^{n} \\
& +\sum_{n=1}^{\infty} \bar{\lambda}_{n}^{B}(\kappa)\left(-\frac{(\nu / 2)^{2 /(2-a)}}{Q^{2}}\right)^{n}
\end{aligned}
$$

The new nonperturbative parameters defining the subleading shape function $\tilde{g}$ are given by expressions similar to Eq. (8),

$$
\begin{aligned}
\bar{\lambda}_{n}^{A}(\kappa, a) & =-\frac{2}{n n !} \int_{0}^{\kappa^{2}} \frac{d p_{\perp}^{2}}{p_{\perp}^{2}}\left(p_{\perp}^{2}\right)^{(2-a) n / 2} A\left(\alpha_{s}\left(p_{\perp}^{2}\right)\right) \\
\bar{\lambda}_{n}^{B}(\kappa) & =\frac{1}{n !} \int_{0}^{\kappa^{2}} \frac{d p_{\perp}^{2}}{p_{\perp}^{2}}\left(p_{\perp}^{2}\right)^{n / 2} B\left(\alpha_{s}\left(p_{\perp}^{2}\right)\right)
\end{aligned}
$$

We notice that for both kinds of subleading contributions there is no simple scaling behavior with $a$. Furthermore, both contributions are suppressed at large $\nu$ (that is, small $\tau_{a}$ ), with an increasing degree of suppression as a grows large and negative: specifically, subleading power corrections appear as functions only of the combination $\nu / Q^{2-a}$, a feature that will also be found in the DGE formalism.

In the following, we will study leading and subleading power corrections by means of DGE, and we will compare the results obtained with those arising directly from the resummation described above. 


\section{Dressed gluon exponentiation for angularities}

Dressed gluon exponentiation begins with a conventional renormalon analysis of the given event shape. One computes the characteristic function of the dispersive approach [12, that is, the contribution to the cross section of a single gluon dressed with an arbitrary number of quark bubbles. This is referred to as the "single dressed gluon" (SDG) cross section. One proceeds by identifying the terms in the characteristic function which contribute to logarithmic behavior in the two-jet limit. These terms can be exponentiated, resumming all contributions where any number of dressed gluons is emitted without interfering. DGE reproduces resummation at NLL, provided the running coupling is defined in the bremsstrahlung scheme. Furthermore, all subleading logs are accounted for in the large $\beta_{0}$ (large $n_{f}$ ) limit. This way, DGE can detect the factorial growth of the coefficients of subleading logarithms, and provide methods to gauge the range of applicability of conventional Sudakov resummations. Furthermore, given an explicit

representation of the singular behavior of perturbation theory, one may pick a definite prescription to deal with the resulting ambiguities.

\subsection{Angularities with a single dressed gluon}

Summing up infinitely many bubbles in the gluon propagator in the inclusive approximation is equivalent to performing a calculation with an off-shell gluon with virtuality $k^{2}$, and replacing the coupling with an effective, "timelike coupling" 30]. One then expresses the SDG cross section by

$$
\left.\frac{1}{\sigma_{\mathrm{tot}}} \frac{d \sigma}{d \tau_{a}}\right|_{\mathrm{SDG}}=\frac{C_{F}}{2 \beta_{0}} \int_{0}^{1} \frac{d \epsilon}{\epsilon} \bar{A}_{\mathrm{eff}}\left(\epsilon Q^{2}\right) \dot{\mathcal{F}}\left(\tau_{a}, \epsilon\right) .
$$

Here $\dot{\mathcal{F}}$ is the derivative with respect to the gluon virtuality $\epsilon=k^{2} / Q^{2}$ of the "characteristic function" $\mathcal{F}$,

$$
\begin{aligned}
\dot{\mathcal{F}}\left(\tau_{a}, \epsilon\right) & =-\epsilon \frac{\partial}{\partial \epsilon} \mathcal{F}\left(\tau_{a}, \epsilon\right), \\
\mathcal{F}\left(\tau_{a}, \epsilon\right) & =\int d x_{1} d x_{2}\left|M\left(x_{1}, x_{2}, \epsilon\right)\right|^{2} \delta\left(\tau_{a}-\tau_{a}\left(x_{1}, x_{2}, \epsilon\right)\right) .
\end{aligned}
$$

The squared matrix element $|M|^{2}$ for the emission of a gluon of virtuality $k^{2}$ in the process $\gamma^{*} \rightarrow q \bar{q} g$, without coupling and color prefactors, is given by

$$
\left|M\left(x_{1}, x_{2}, \epsilon\right)\right|^{2}=\frac{\left(x_{1}+\epsilon\right)^{2}+\left(x_{2}+\epsilon\right)^{2}}{\left(1-x_{1}\right)\left(1-x_{2}\right)}-\frac{\epsilon}{\left(1-x_{1}\right)^{2}}-\frac{\epsilon}{\left(1-x_{2}\right)^{2}} .
$$

$x_{1}$ and $x_{2}$, as usual, are the energy fractions of the quark and antiquark in the centerof-mass frame, and one can define $x_{3}=2-x_{1}-x_{2}$, the gluon energy fraction. When 
the gluon is "massive", the limits of phase space are given by

$$
\begin{aligned}
x_{1}+x_{2} & \geq 1-\epsilon \\
\left(1-x_{1}\right)\left(1-x_{2}\right) & \geq \epsilon .
\end{aligned}
$$

The final ingredient in Eq. (13) is the "timelike coupling" $\bar{A}_{\text {eff }}$, which is typically expressed in terms of a Borel representation as

$$
\bar{A}_{\mathrm{eff}}\left(Q^{2}\right)=\int_{0}^{\infty} d u\left(\frac{Q^{2}}{\Lambda_{\mathrm{QCD}}^{2}}\right)^{-u} \frac{\sin \pi u}{\pi u} \bar{A}_{B}(u) .
$$

We now need to specify a suitable generalization of the massless definition of the event shape, Eq. (11), for the case in which the emitted gluon has nonvanishing virtuality. Several observations are helpful in deciding how to perform this generalization. First, all logarithmic contributions to the SDG cross sections stem from the region where the gluon is either soft or collinear. Contributions from the region where the gluon is relatively hard do not give logarithmic enhancements, and the exact location of the boundary between the region of phase space where one of the quarks is dominant and the region where the gluon dominates is unimportant. We can thus concentrate on the phase space region where the thrust axis is the quark momentum, and $x_{1}$ the largest energy fraction. Contributions in which the antiquark momentum dominates can be obtained by symmetry. Recall the expression for $\tau_{a}$ in the case of massless partons, in the region where the quark has the largest energy,

$$
\tau_{a}\left(x_{1}, x_{2}\right)=\frac{\left(1-x_{1}\right)^{1-a / 2}}{x_{1}}\left[\left(1-x_{2}\right)^{1-a / 2}\left(x_{1}+x_{2}-1\right)^{a / 2}+\left(x_{1} \leftrightarrow x_{2}\right)\right] .
$$

We would like our definition to reduce to Eq. (18) as $\epsilon \rightarrow 0$. Furthermore, we require that for $a=0$ the definition should reduce to the massive definition of the thrust, as used for example in [13, 17, 18, 25]. In the phase space region at hand, this is simply $\tau_{0}\left(x_{1}, x_{2}, \epsilon\right)=1-x_{1}$. Finally, as we will see, working analytically for generic $a$ and with a massive gluon generates rather intricate expressions, so we must keep the definition as simple as possible in order to be able to perform the necessary integrations.

Keeping these criteria in mind, we define the angularity with an off-shell gluon, in the region where the thrust axis is given by the quark momentum, as

$$
\begin{aligned}
\tau_{a}\left(x_{1}, x_{2}, \epsilon\right)=\frac{\left(1-x_{1}\right)^{1-a / 2}}{x_{1}} & {\left[\left(1-x_{2}-\epsilon\right)^{1-a / 2}\left(x_{1}+x_{2}-1+\epsilon\right)^{a / 2}\right.} \\
+ & \left.\left(x_{1}+x_{2}-1+\epsilon\right)^{1-a / 2}\left(1-x_{2}-\epsilon\right)^{a / 2}\right] .
\end{aligned}
$$

Of course, other choices satisfying our criteria are possible. It can be shown, however, that different treatments of the gluon mass alter the value of $\tau_{a}$ by terms that are 
suppressed by higher powers of the weight, roughly by a factor of $\tau_{a}^{1-a}$. Thus, they do not change logarithmically enhanced contributions. Once a definite prescription to include massive partons is chosen, the predictions within the DGE formalism are unambiguous. It should be kept in mind, in any case, that a comparison to experiment requires a detailed analysis along the lines of Ref. 28 for the inclusion of hadron mass effects.

To proceed, it is useful to change integration variables from $x_{1}, x_{2}$ to

$$
\begin{aligned}
\zeta & =1-x_{1} \\
\xi & =\frac{x_{1}+x_{2}-1+\epsilon}{1-x_{2}-\epsilon} .
\end{aligned}
$$

In terms of these variables, the characteristic function can be written as

$$
\begin{aligned}
\mathcal{F}\left(\tau_{a}, \epsilon\right)=\int_{\epsilon}^{\sqrt{\epsilon}} d \zeta \int_{\frac{\zeta}{1-2 \zeta}}^{\frac{\zeta}{\epsilon}-1} d \xi\left[\frac{(1-\zeta+\epsilon)^{2}(1-\zeta)}{\zeta(1-\zeta+\epsilon(1+\xi))(1+\xi)}\right. \\
\quad+\frac{(\xi+\zeta)^{2}(1-\zeta)}{\zeta(1-\zeta+\epsilon(1+\xi))(1+\xi)^{3}}-\frac{\epsilon(1-\zeta)}{(1-\zeta+\epsilon(1+\xi))^{2}} \\
\left.\quad-\frac{\epsilon(1-\zeta)}{\zeta^{2}(1+\xi)^{2}}\right] \delta\left(\tau_{a}-\frac{\zeta^{1-a / 2}}{1+\xi} \xi^{a / 2}\left(1+\xi^{1-a}\right)\right)
\end{aligned}
$$

Two comments are in order. First, the limits of integration do not correspond to the full phase space, but only to the region which generates logarithmically enhanced contribution, that is, the region in which the gluon is either soft or collinear to the quark. With a massive gluon, the collinear limit corresponds to $x_{1}=1-\epsilon, x_{2}=0$, whereas the soft limit is given by $x_{1}=x_{2}=1-\sqrt{\epsilon}$. These two values set the limits of the $\zeta$ integration. The soft boundary of phase space for intermediate values of $\zeta$ is given by $x_{2} \leq 1-\epsilon /\left(1-x_{1}\right)$, corresponding to the upper limit of the $\xi$ integration. The Sudakov region thus corresponds to values of $\xi$ close to the upper boundary of integration. The second observation is that, with this choice for angularity in the presence of a massive gluon, and in these variables, the $\delta$-function defining the curves of constant angularity in Eq. (21) does not depend on $\epsilon$, a feature that will be exploited below.

\subsection{Exponentiation and Borel representation}

The SDG cross section summarizes the probability that one dressed gluon is emitted. Upon summing over probabilities that many such dressed gluons are emitted independently, one obtains the exponentiated expression [17, 18]

$$
\left.\frac{1}{\sigma_{\mathrm{tot}}} \tilde{\sigma}(\nu, a)\right|_{\mathrm{DGE}}=\exp \left[\int_{0}^{\infty} d \tau_{a}\left(e^{-\nu \tau_{a}}-1\right)\left(\left.\frac{1}{\sigma_{\mathrm{tot}}} \frac{d \sigma}{d \tau_{a}}\right|_{\mathrm{SDG}}\right)\right] .
$$


Here we have extended the integration region beyond the support of the SDG cross section. This, however, does not change the result in the region of interest, at small $\tau_{a}$ or equivalently at large $\nu$. Inserting Eq. (13) and Eq. (17) into Eq. (22), one may write down an explicit Borel representation for the exponent, although at this point one is still dealing with a five-fold integral. One finds

$\ln \left[\frac{1}{\sigma_{\mathrm{tot}}} \tilde{\sigma}(\nu, a)\right]_{\mathrm{DGE}}=\frac{C_{F}}{2 \beta_{0}} \int_{0}^{\infty} d u\left(\frac{Q^{2}}{\Lambda_{\mathrm{QCD}}^{2}}\right)^{-u} \frac{\sin \pi u}{\pi u} \bar{A}_{B}(u) \int_{0}^{\infty} \frac{d \epsilon}{\epsilon} \epsilon^{-u} \int_{\tau_{a}^{\mathrm{co}}}^{\tau_{a}^{\mathrm{s}}} d \tau_{a}\left(e^{-\nu \tau_{a}}-1\right) \dot{\mathcal{F}}\left(\tau_{a}, \epsilon\right)$.

To exchange the order of integration, as done in Eq. (23), it has been necessary to evaluate the limiting values of angularity in the phase space region of interest, for a fixed gluon virtuality $\epsilon$. One finds that for any value of $a$ the collinear limit of angularity is given by $\tau_{a}^{\mathrm{co}}=\epsilon+\mathcal{O}\left(\epsilon^{2}\right)$, while the soft limit is $\tau_{a}^{\mathrm{s}}=\sqrt{\epsilon}+\mathcal{O}(\epsilon)$ (notice that the corrections neglected here vanish for $a=0$ ). As shown below, however, the exact form of these limits is not important: only the values of the energy fractions of the quark and of the gluon at the soft and collinear boundaries of phase space are relevant, and they are independent of the weight $\tau_{a}$, and solely dependent on the kinematics.

The advantage of writing the exponent as in Eq. (23) is that it is now possible, before performing phase space integrals, to take the derivative with respect to $\epsilon$ of Eq. (21), thanks to the fact that the $\delta$-function defining $\tau_{a}$ does not depend on $\epsilon$ with our choice of variables. One may then use the $\delta$-function to perform trivially the $\tau_{a}$ integral. Discarding terms that do not contribute Sudakov logarithms, we can write the Borel representation of the exponent as

$$
\ln \left[\frac{1}{\sigma_{\mathrm{tot}}} \tilde{\sigma}(\nu, a)\right]_{\mathrm{DGE}}=\frac{C_{F}}{2 \beta_{0}} \int_{0}^{\infty} d u\left(\frac{Q^{2}}{\Lambda_{\mathrm{QCD}}^{2}}\right)^{-u} \frac{\sin \pi u}{\pi u} \bar{A}_{B}(u) B(u, \nu, a),
$$

with the Borel function $B(u, \nu, a)$ given by

$$
\begin{aligned}
B(u, \nu, a) & \equiv \int_{0}^{\infty} d \epsilon \epsilon^{-u} \int_{\epsilon}^{\sqrt{\epsilon}} d \zeta\left\{\int_{0}^{\zeta / \epsilon-1} d \xi \frac{1}{\zeta^{2}(1+\xi)^{2}}\left[\exp \left(-\nu \zeta^{(2-a) / 2} \frac{\xi^{a / 2}}{1+\xi}\left(1+\xi^{1-a}\right)\right)-1\right]\right. \\
& \left.+\left(\frac{2}{\epsilon \zeta}-\frac{2}{\zeta^{2}}\right)\left[\exp \left\{-\nu \epsilon \zeta^{-a / 2}\left(\frac{\zeta}{\epsilon}-1\right)^{a / 2}\left(1+\left(\frac{\zeta}{\epsilon}-1\right)^{1-a}\right)\right\}-1\right]\right\} \cdot(25)
\end{aligned}
$$

The factors of -1 in Eq. (25) are due to virtual corrections. They contribute terms independent of $a$, and are thus identical to the thrust [17, 18]. They do not need to be considered anew. We will now concentrate on the contributions associated with real gluon emission, labelled in the following by an additional subscript, $B_{R}(u, \nu, a)$. 
It is possible to arrive at a one-dimensional integral representation of $B_{R}(u, \nu, a)$. First we perform the integration over $\zeta$ in the first term of Eq. (25) , and change variables from $\zeta$ to $\xi=\zeta / \epsilon-1$ in the second term. Finally, integrating over $\epsilon$ results in the following expression

$$
\begin{aligned}
B_{R}(u, \nu, a)= & -\frac{1}{u} \int_{0}^{\infty} d \xi(1+\xi)^{2 u}\left[\frac{2}{1+\xi}-\frac{2}{(1+\xi)^{2}}+\frac{1}{1-u} \frac{1}{(1+\xi)^{3}}\right] \\
& \times{ }_{1} F_{1}\left[-\frac{2 u}{2-a} ; 1-\frac{2 u}{2-a} ;-\nu \xi^{a / 2} \frac{1+\xi^{1-a}}{(1+\xi)^{2-a / 2}}\right]+\mathcal{O}\left(\frac{1}{\nu}\right),
\end{aligned}
$$

where ${ }_{1} F_{1}$ is the confluent hypergeometric function, also known as Kummer's function of the first kind, defined by [31]

$$
{ }_{1} F_{1}(\alpha ; \beta ; z) \equiv \sum_{k=0}^{\infty} \frac{(\alpha)_{k}}{(\beta)_{k}} \frac{z^{k}}{k !},
$$

where the Pochhammer symbol is defined as

$$
(\alpha)_{k} \equiv \alpha(\alpha+1) \ldots(\alpha+k-1)=\frac{\Gamma(\alpha+k)}{\Gamma(\alpha)} .
$$

Clearly, Eq. (26) cannot be evaluated directly. Nevertheless, in the physically interesting limits, the soft and collinear region of phase space, results in closed form can be obtained, and from these results the form of power corrections can be inferred. Before discussing the results for general $a$, however, we pause to note that we can test Eq. (26) by considering the case of the thrust, $a=0$, where the answer is known [17, 18]. For $a=0$, indeed, Eq. (26) can be explicitly integrated. From the resulting expressions one can see that only the factor $2 /(1+\xi)$ in the square bracket of Eq. (26) contributes in the soft limit, whereas the remaining contributions are of purely collinear origin. The final result for $B_{R}(u, \nu, 0)$ is

$$
B_{R}(u, \nu, 0)=\nu^{2 u} \Gamma(-2 u) \frac{2}{u}-\nu^{u} \Gamma(-u)\left(\frac{2}{u}+\frac{1}{1-u}+\frac{1}{2-u}\right),
$$

up to corrections of order $1 / \nu$. Our result is in complete agreement with Refs. [17, 18]. Soft contributions produce singularities at half-integer values of $u$, corresponding to power corrections of the form $\left(\Lambda_{\mathrm{QCD}} \nu / Q\right)^{p}$; collinear contributions have poles at integer $u$, giving power corrections behaving as $\left(\Lambda_{\mathrm{QCD}}^{2} \nu / Q^{2}\right)^{p}$. We note also that the result in Eq. (29) is infrared safe at leading power. In other words, the poles at $u=0$ (corresponding to $Q^{0}$ ) cancel between the soft and the collinear contributions. We will verify this key property of infrared safety also for general $a$. 


\section{The pattern of exponentiated power corrections}

\subsection{Power corrections from soft radiation}

We now turn to the soft contribution for general $a$, which is the source of the scaling in Eq. (91). In the region of phase space corresponding to soft emission one has $\zeta \sim \sqrt{\epsilon}$, so that $\xi$ is large, behaving as $1 / \sqrt{\epsilon}$. In this region, we will first evaluate Eq. (26) by making use of an asymptotic expansion for the Kummer function. Then we will go back to the characteristic function $\mathcal{F}\left(\tau_{a}, \epsilon\right)$ and evaluate it in the same limit, to trace the contributions that display scaling back to the matrix element.

To treat the soft limit, we can use the following integral representation of the confluent Kummer function [32],

$$
{ }_{1} F_{1}(\alpha ; \beta ; z)=\frac{\Gamma(\beta)}{\Gamma(\alpha) \Gamma(\beta-\alpha)} \int_{0}^{1} d t e^{z t} t^{\alpha-1}(1-t)^{-\alpha+\beta-1} .
$$

We then see that the complicated argument of the Kummer function in Eq. (26) appears

only in the exponent, and can be expanded for large $\xi$ and $\nu$, with $\nu / \xi$ held fixed. One finds

$$
\exp \left[-\nu t \xi^{a / 2} \frac{1+\xi^{1-a}}{(1+\xi)^{2-a / 2}}\right]=\exp \left[-\frac{\nu t}{\xi}\right]+\ldots .
$$

The soft contribution then simplifies considerably, and becomes

$$
\begin{aligned}
B_{R}^{\text {soft }}(u, \nu, a)= & \frac{2}{2-a} \int_{0}^{\infty} d \xi \int_{0}^{1} d t e^{-\nu t / \xi} t^{-\frac{2 u}{2-a}-1}(1+\xi)^{2 u} \\
& \times\left[\frac{2}{1+\xi}-\frac{2}{(1+\xi)^{2}}+\frac{1}{1-u} \frac{1}{(1+\xi)^{3}}\right]+\mathcal{O}\left(\frac{1}{\nu}\right) .
\end{aligned}
$$

Since we are interested in the large $\xi$ limit, clearly at leading power only the first term in the bracket of Eq. (32) contributes, while the remaining two terms are suppressed by powers of $1 / \nu$. The integral can be performed, and one finds that the final answer for the soft contribution is

$$
B_{R}^{\text {soft }}(u, \nu, a)=\frac{1}{1-a} \nu^{2 u} \Gamma(-2 u) \frac{2}{u}+\mathcal{O}\left(\frac{1}{\nu}\right) .
$$

Comparing this with the case for $a=0$, Eq. (29), we see that we recover exactly the prediction of Eq. (92). We have thus confirmed the result of [23], obtained there by analyzing the resummed expression. 


\subsection{An alternative derivation of the scaling rule}

To perform the integrations leading to Eq. (33) for generic $a$, it has been necessary to make use of some special function technology. This technology may obscure the physics underlying the simple scaling, and indeed the result of Eq. (33) may appear somewhat surprising, in view of the fact that, for example, Eq. (32) still has an overall factor of $2 /(2-a)$, corresponding to the scaling of leading logarithms but not to the scaling of power corrections. It is thus instructive to recover the result by going back to the characteristic function, Eq. (21), and identifying the origin of the terms responsible for the leading soft contribution.

The first observation to simplify Eq. (21) concerns phase space. As observed before, the Sudakov region corresponds to a neighborhood of the upper limit of the $\xi$ integration range, whereas the lower limit does not contribute to logarithmic enhancements and can be changed. To simplify the calculation, we can for example take as a lower limit $\xi=0$, as was already done in Eq. (25). The expression for $\mathcal{F}$ becomes then slightly more manageable by changing variables from $\xi$ to $\omega=1+\xi$. One finds

$$
\begin{aligned}
\mathcal{F}\left(\tau_{a}, \epsilon\right)= & \int_{1}^{1 / \sqrt{\epsilon}} d \omega \int_{\epsilon \omega}^{\sqrt{\epsilon}} d \zeta \frac{1-\zeta}{\zeta}\left[\frac{(1-\zeta+\epsilon)^{2}}{\omega(1-\zeta+\epsilon \omega)}+\frac{(\zeta+\omega-1)^{2}}{\omega^{3}(1-\zeta+\epsilon \omega)}\right. \\
& \left.-\frac{\epsilon}{\zeta \omega^{2}}-\frac{\epsilon \zeta}{(1-\zeta+\epsilon \omega)^{2}}\right] \delta\left(\tau_{a}-\zeta^{1-a / 2} f_{a}(\omega)\right),
\end{aligned}
$$

where we define

$$
f_{a}(\omega)=(\omega-1)^{a / 2} \frac{1+(\omega-1)^{1-a}}{\omega} .
$$

Notice that $f_{0}(\omega)=1$, which of course simplifies things considerably for the thrust. For any $a$, the $\zeta$ dependence in the $\delta$-function is sufficiently simple to be used to perform the $\zeta$ integration, yielding a rather cumbersome expression, which however has some interesting features. One finds

$$
\begin{aligned}
\mathcal{F}\left(\tau_{a}, \epsilon\right)= & \frac{2}{2-a} \frac{1}{\tau_{a}} \int_{1}^{1 / \sqrt{\epsilon}} \frac{d \omega}{\omega}\left(1-\bar{\zeta}_{a}\right)\left[\frac{\left(1-\bar{\zeta}_{a}+\epsilon\right)^{2}}{\left(1-\bar{\zeta}_{a}+\epsilon \omega\right)}+\frac{\left(\bar{\zeta}_{a}+\omega-1\right)^{2}}{\omega^{2}\left(1-\bar{\zeta}_{a}+\epsilon \omega\right)}\right. \\
& \left.-\frac{\epsilon}{\bar{\zeta}_{a} \omega}-\frac{\epsilon \bar{\zeta}_{a} \omega}{\left(1-\bar{\zeta}_{a}+\epsilon \omega\right)^{2}}\right] \theta\left(\bar{\zeta}_{a}-\epsilon \omega\right) \theta\left(\sqrt{\epsilon}-\bar{\zeta}_{a}\right),
\end{aligned}
$$

where

$$
\bar{\zeta}_{a}=\left(\frac{\tau_{a}}{f_{a}(\omega)}\right)^{\frac{2}{2-a}}=\frac{\left(\omega \tau_{a}\right)^{\frac{2}{2-a}}(\omega-1)^{-\frac{a}{2-a}}}{\left[1+(\omega-1)^{1-a}\right]^{\frac{2}{2-a}}} .
$$

Several observations help disentangling Eq. (36). First, the second $\theta$-function is not relevant to the logarithmic behavior and can be neglected. Its effect would be to split 
the integration range for $\omega$ in two subintervals, however all leading contributions come from neighborhoods of the upper and lower limits of integration. A second important point is the fact that the leading singularity in $\tau_{a}$ is now explicitly factored out. One can then evaluate the integral to leading power in $\tau_{a}$. The constraint imposed by the first $\theta$-function can also be considerably simplified. The integral, in fact, has support on the region defined by

$$
\frac{\tau_{a}}{\epsilon^{1-a / 2}} \geq\left(\frac{\omega}{\omega-1}\right)^{-a / 2}\left[1+(\omega-1)^{1-a}\right] .
$$

This constraint cannot be solved exactly for $\omega$. One notices, however, that in the physical region, $\epsilon \leq \tau_{a} \leq \sqrt{\epsilon}$, and for $a \leq 0$, the left hand side of Eq. (38) is a parametrically large number. Within the integration range, on the other hand, the right hand side becomes large only near the boundaries, as $\omega \rightarrow \infty$ or as $\omega \rightarrow 1$. One can then solve the $\theta$-function constraint in these two limits, obtaining respectively

$$
\omega<\omega_{+} \equiv\left(\frac{\tau_{a}}{\epsilon^{1-a / 2}}\right)^{\frac{1}{1-a}}, \quad \omega \rightarrow \infty
$$

and

$$
\omega>\omega_{-} \equiv 1+\frac{\epsilon^{1-2 / a}}{\tau_{a}^{-2 / a}}, \quad \omega \rightarrow 1
$$

The result of these manipulations is that the integration region in Eq. (36) shrinks at both boundaries, with both integration limits now dependent on $\tau_{a}$. Since soft contributions arise from the region of large $\omega$, we will concentrate on Eq. (39), although similar arguments could be used with Eq. (40). This will be sufficient to recover the scaling rule.

To complete the calculation, we must now approximate the matrix element. To this end, note that for large $\omega$ one can approximate Eq. (37) by

$$
\bar{\zeta}_{a}=\tau_{a}^{\frac{2}{2-a}} \omega^{\frac{a}{2-a}} \ll 1
$$

One sees that $\bar{\zeta}_{a}$ is a small number in the relevant range of $a$, and we can expand the integrand of Eq. (36) around $\bar{\zeta}_{a}=0$. The resulting soft approximation of the characteristic function is

$$
\left.\mathcal{F}\left(\tau_{a}, \epsilon\right)\right|_{\text {soft }}=\frac{2}{2-a} \frac{1}{\tau_{a}} \int^{\omega_{+}} \frac{d \omega}{\omega}\left[2-\frac{2}{\omega}+\frac{1}{\omega^{2}}-\frac{\epsilon}{\left(\tau_{a} \omega\right)^{\frac{2}{2-a}}}\right] .
$$

The integration can now be performed and compared with the result for the thrust. At large $\omega$ the dominant contribution clearly comes from the first term in the square bracket. Comparing with the case $a=0$, we see in fact that, while all terms contribute to the Sudakov limit, the last three terms give subleading corrections associated with 
collinear radiation. This remains true for generic $a$, as noted in the following subsection. Concentrating on the first term, we can finally see how the scaling rule arises in the context of DGE. The only term in the cross section contributing in the soft limit is, as might be expected, the logarithmic integral over the bremsstrahlung gluon spectrum. The gluon mass then acts as an infrared cutoff on this integral. The power of $\epsilon$ in the upper limit of integration $\omega_{+}$is precisely the one required to cancel the overall factor of $2 /(2-a)$, and to replace it with the scaling factor of Eq. (9). This precise power arises uniquely from the definition of angularity, as expressed by the $\delta$-function in Eq. (34), and can easily be traced back to the exponential weight given to pseudorapidity in Eq. (10). The final result in the soft limit is simply

$$
\left.\mathcal{F}\left(\tau_{a}, \epsilon\right)\right|_{\text {soft }}=-\frac{1}{1-a} \frac{2}{\tau_{a}} \ln (\epsilon),
$$

where subleading collinear contributions and terms independent of $\epsilon$, which do not contribute to the logarithmic behavior, have been omitted. Power corrections of collinear origin can be treated similarly, giving results consistent with the ones outlined in the following subsection.

\subsection{Power corrections from collinear radiation}

At this point we have various methods at our disposal to analyze power corrections of collinear origin. We can for example complete the analysis of Section 4.2, including the effects of the lower limit of integration, and then inserting the results in the Borel exponent. Alternatively, we can go back to Eq. (26) and study it in this limit. Collinear power corrections arise at large $\nu$, but small $\xi$. In this limit, Eq. (26) yields

$$
\begin{aligned}
B_{R}^{\text {coll. }}(u, \nu, a)= & \frac{2}{2-a} \Gamma\left(-\frac{2 u}{2-a}\right) \nu^{\frac{2 u}{2-a}} \int_{0}^{\infty} d \xi \xi^{\frac{a}{2-a} u}(1+\xi)^{-\frac{a}{2-a} u}\left(1+\xi^{1-a}\right)^{\frac{2 u}{2-a}} \\
& \times\left[\frac{2}{1+\xi}-\frac{2}{(1+\xi)^{2}}+\frac{1}{1-u} \frac{1}{(1+\xi)^{3}}\right]+\mathcal{O}\left(\frac{1}{\nu}\right) .
\end{aligned}
$$

We see immediately that collinear contributions are suppressed by non-integer powers compared to the leading soft piece, as found above in the derivation of Eq. (9). This non-integer power behavior can also be seen from Eq. (44), which has singularities at non-integer $u$ due to the $\Gamma$-function, and further singularities due to the integration over $\xi$, which can be readily performed for any specific value of $a$. For generic $a$, the leading poles on the positive real $u$ axis can also be determined by studying the integrand of Eq. (44) in the limits of large and small $\xi$. Determining the full analytic structure of Eq. (44) as a function of $a$ is however considerably more difficult. In the case when $a$ is a rational number, we have been able to obtain a closed expression in terms of generalized 
hypergeometric functions ${ }_{p} F_{q}$. The detailed expressions are listed in Appendix A. Since all methods give consistent results, we will just summarize here the structure of poles on the positive real axis of the Borel variable $u$, and we will outline the corresponding pattern of power corrections.

First, let us note that our complete result for the Borel function $B$ is infrared safe, as it must. In fact, as was the case for the thrust, the poles at $u=0$ cancel between soft and collinear contributions. To see this, note for example that the intricate expression in Eq. (58) simplifies at $u=0$ to

$$
B_{R}^{\text {coll. }}(u \sim 0, \nu, a)=\nu^{\frac{2}{2-a} u} \Gamma\left(-\frac{2}{2-a} u\right) \frac{4}{2-a} \Gamma\left(-\frac{2(1-a)}{2-a} u\right),
$$

where we have suppressed terms nonsingular at $u=0$. This clearly cancels against the contribution of Eq. (33) at $u=0$, as expected.

Next, we note that Eq. (44) appears to have an explicit pole at $u=1$, which would correspond to a correction of order $\mathcal{O}\left(\nu\left(\Lambda_{\mathrm{QCD}} / Q\right)^{2}\right)$. This pole, however, is cancelled by the explicit factor of $\sin \pi u$ in Eq. (23), as was the case for the thrust (where power corrections of this form are present only as an effect of the $\Gamma$-functions in Eq. (29)).

The general structure of poles in $u$, for rational $a$, can be deduced from Eq. (58) in Appendix A. There are several infinite towers of poles. From Eq. (44) we can directly read off the first pole in each tower. We find singularities at

$$
\begin{aligned}
& u=u_{1}=\frac{2-a}{2}, \\
& u=u_{2}=-\frac{2-a}{a}, \\
& u=u_{3}=\frac{1}{2} \frac{2-a}{1-a} .
\end{aligned}
$$

Recall that $a \leq 0$ in the range of validity of our approach. The crucial fact here is that all these singularities are accompanied by a common factor of $\nu^{2 u /(2-a)}$, as seen in Eq. (44). When combined with the locations of the poles in Eq. (46), we see that all collinear power corrections are expressed in terms of a single combination of $\nu$ and of the scale $Q$, namely $\nu\left(\Lambda_{\mathrm{QCD}} / Q\right)^{2-a}$, possibly further raised to a non-integer power. This result agrees with the estimate extracted for the resummation, Eqs. (10)-(11), although the detailed pattern of subleading singularities is different. We conclude that collinear power corrections are suppressed as predicted from resummation, and are expected to become important only for extreme values of the angularity $\tau_{a}, \tau_{a} \sim \mathcal{O}\left(\Lambda_{\mathrm{QCD}} / Q\right)^{2-a}$. The suppression grows as $a$ becomes large and negative, although numerically the effect could be partly compensated by the fact some of the corrections may be further raised to small non-integer powers, of order $1 /(1-a)$. 
It is also important to note that the intricate structure of towers of subleading poles arising from Eq. (58) is not as model-independent as the leading poles connected to soft radiation. For example, a different choice for the massive definition of angularity, Eq. (19), might kinematically generate non-integer power corrections of comparable size. We emphasize, in any case, that the leading structure expressed by Eq. (133) is unaffected. Furthermore, we believe that the parametric dependence of collinear power corrections on the ratio $\nu\left(\Lambda_{\mathrm{QCD}} / Q\right)^{2-a}$, which is found via resummation, and confirmed by our DGE analysis, is a stable feature, suggesting that the scaling rule should hold with increasing precision for negative $a$.

\section{Conclusions}

In this work we have verified that the universal scaling of the leading power corrections within the class of angularities, discovered in Ref. [23] from soft gluon resummation, is unchanged when, in addition to large Sudakov logarithms, also renormalon enhancements are taken into account. Furthermore, we have been able to determine the form of subleading corrections within a specific scheme to treat massive partons. These corrections, of collinear origin, are suppressed by non-integer powers of the hard scale relative to the leading ones that originate from soft radiation. Different models of these collinear corrections further suggest that the relative suppression grows as the parameter $a$ becomes large and negative. A detailed phenomenological study along the lines of Refs. [17, 18. could help to further constrain the nonleading effects, and would be necessary for a comparison with experiment. We defer such a study to future work, due to the lack of corresponding experimental data.

Assuming subleading corrections are negligible, as indeed our results imply, the scaling allows to predict the distributions of the whole class of angularities, including nonperturbative corrections, in terms of a single shape function, which can in principle be determined from data by considering a specific choice of the parameter $a$, for example the thrust, $a=0$. Since there are no free parameters, an experimental determination

of distributions for other values of $a$ would certainly give valuable information about the properties of nonperturbative corrections. Also, since the perturbative cross section has a scaling behavior with $a$ different from the non-perturbative shape function, comparison of theory and experiment might help to disentangle corrections due to missing higher-order perturbative information from power correction effects. An experimental test of scaling would determine to what extent the boost invariance of soft radiation in the two-jet limit, which is established at the perturbative level, also applies to nonperturbative effects. We hope therefore that an analysis of experimental data for the class of angularities will be made in the near future. 


\section{A Evaluation of the collinear contribution}

Here we sketch the evaluation of the collinear contribution to the DGE cross section for rational $a$, that is, we consider the case

$$
1-a=\frac{p}{q}, \quad p, q \text { positive integer, } p \geq q .
$$

This is sufficient for our purposes, since any irrational number can always be well approximated by a rational one.

The collinear limit is equivalent to the limit in which $\nu \rightarrow \infty$ faster than $\xi$ (although $\xi$ varies between 0 and $\infty$ ). In this limit the collinear contribution is given by Eq. (44). In the following we will abbreviate Eq. (44) by

$$
B_{R}^{\text {coll. }}(u, \nu, a)=\frac{2}{2-a} \Gamma\left(-\frac{2 u}{2-a}\right) \nu^{\frac{2 u}{2-a}} \sum_{m=1}^{3} c_{m} \mathcal{I}^{m},
$$

where

$$
\begin{aligned}
\mathcal{I}^{m} & =\int_{0}^{\infty} d \xi \xi^{\frac{a}{2-a} u}(1+\xi)^{-\frac{a}{2-a} u-m}\left(1+\xi^{1-a}\right)^{\frac{2 u}{2-a}} \\
c_{1} & =2, \quad c_{2}=-2, \quad c_{3}=\frac{1}{1-u} .
\end{aligned}
$$

We use the Mellin-Barnes representation of $\left(1+\xi^{1-a}\right)^{2 u /(2-a)}, a=1-p / q$,

$$
\left(1+\xi^{p / q}\right)^{2 q u /(p+q)}=\frac{1}{2 \pi i} \int_{C} d \alpha \xi^{\alpha p / q} \frac{\Gamma(-\alpha) \Gamma\left(\alpha-\frac{2 q u}{p+q}\right)}{\Gamma\left(-\frac{2 q u}{p+q}\right)} .
$$

The contour $C$ runs along the imaginary axis, to the left of $\operatorname{Re}(\alpha)=0$. With this we can rewrite Eq. (49) in terms of $p$ and $q$ as

$$
\mathcal{I}^{m}=\int_{C} \frac{d \alpha}{2 \pi i} \frac{\Gamma(-\alpha) \Gamma\left(\alpha-\frac{2 q}{q+p} u\right)}{\Gamma\left(-\frac{2 q}{q+p} u\right)} \frac{\Gamma\left(m-1-\frac{p}{q} \alpha\right) \Gamma\left(1+\frac{(q-p)}{q+p} u+\frac{p}{q} \alpha\right)}{\Gamma\left(m+\frac{(q-p)}{q+p} u\right)} .
$$

We change variables from $\alpha$ to $\tilde{\alpha}=\alpha / q$, with $\tilde{C}$ the contour in the new variable, and use the following properties of the $\Gamma$-function [31, 32]:

$$
\begin{aligned}
& \Gamma(n z)=n^{n z-1 / 2}(2 \pi)^{(1-n) / 2} \prod_{k=0}^{n-1} \Gamma\left(z+\frac{k}{n}\right), \quad n \text { integer } \\
& \Gamma(z-n)= \frac{(-1)^{n} \Gamma(z)}{(1-z)_{n}} \\
& \operatorname{Res}_{\alpha} \quad\{\Gamma(\alpha-b) f(\alpha)\}=\sum_{n=0}^{\infty} \frac{(-1)^{n}}{n !} f(\alpha=b-n),
\end{aligned}
$$


where in (55) the residues of the $\Gamma$-function are taken with respect to $\alpha$. This allows us to rewrite (52) as

$$
\begin{aligned}
\mathcal{I}^{m}= & \frac{q^{-\frac{2 q u}{q+p}} p^{m-1+\frac{q-p}{q+p} u}(2 \pi)^{2-p-q}}{\Gamma\left(-\frac{2 q}{q+p} u\right) \Gamma\left(m+\frac{q-p}{q+p} u\right)} \int_{\tilde{C}} \frac{d \tilde{\alpha}}{2 \pi i} \prod_{k=0}^{q-1} \Gamma\left(-\tilde{\alpha}+\frac{k}{q}\right) \prod_{i=0}^{p-1} \Gamma\left(-\tilde{\alpha}+\frac{m-1+i}{p}\right) \\
& \quad \times \prod_{j=0}^{q-1} \Gamma\left(\tilde{\alpha}-\frac{2}{q+p} u+\frac{j}{q}\right) \prod_{h=0}^{p-1} \Gamma\left(\tilde{\alpha}+\frac{q-p}{(q+p) p} u+\frac{h+1}{p}\right) .
\end{aligned}
$$

We now close the contour to pick up the residues at $\tilde{\alpha}=\frac{2}{p+q} u-\frac{j}{q}-n, n$ integer, where $j$ runs from 0 to $q-1$, and $\tilde{\alpha}=-\frac{h+1}{m}-\frac{q-p}{(q+p) p} u-n$, where $h$ runs from 0 to $p-1$. We thus have $p+q$ residues, evaluated according to (55). After a bit of algebra, using Eqs. (53) and (154), as well as the definition of the Pochhammer symbol (28), we can rewrite the result in terms of generalized hypergeometric functions, which are defined as [31]

$$
{ }_{p} F_{q}\left(\left\{\alpha_{1}, \ldots, \alpha_{p}\right\} ;\left\{\beta_{1}, \ldots, \beta_{q}\right\} ; z\right) \equiv \sum_{n=0}^{\infty} \frac{z^{n}}{n !} \frac{\prod_{i=1}^{p}\left(\alpha_{i}\right)_{n}}{\prod_{j=1}^{q}\left(\beta_{j}\right)_{n}} .
$$

In order to find the poles in $u$ with respect to $a$, it is instructive to partially rewrite the result in terms of $a$ where possible (recall that $p=(1-a) q)$ :

$$
\begin{aligned}
\mathcal{I}^{m}=\sum_{r=0}^{q-1} & q^{1 / 2-r}(2 \pi)^{\frac{1-q}{2}} \prod_{j=0, j \neq r}^{q-1} \Gamma\left(\frac{j-r}{q}\right) \\
& \times{ }_{(2-a) q} F_{(2-a) q-1}\left(\{\mathcal{A}\} ;\{\mathcal{B}\} ;(-1)^{(2-a) q}\right) \\
& \times \frac{\Gamma\left(r-\frac{2}{2-a} u\right)}{\Gamma\left(-\frac{2}{2-a} u\right)} \frac{\Gamma\left((1-a) r+m-1-\frac{2(1-a)}{2-a} u\right) \Gamma(1+u-(1-a) r)}{\Gamma\left(m+\frac{a}{2-a} u\right)} \\
+\sum_{t=0}^{(1-a) q-1} & q^{1 / 2-t}(1-a)^{-1 / 2-t}(2 \pi)^{\frac{1-(1-a) q}{2}} \prod_{j=0, j \neq t}^{(1-a) q-1} \Gamma\left(\frac{j-t}{(1-a) q}\right) \\
& \times{ }_{(2-a) q} F_{(2-a) q-1}\left(\left\{\mathcal{A}^{\prime}\right\} ;\left\{\mathcal{B}^{\prime}\right\} ;(-1)^{(2-a) q}\right) \\
& \times \frac{\Gamma\left(t+m+\frac{a}{2-a} u\right)}{\Gamma\left(\frac{1}{1-a}(t+1)+\frac{1}{1-a} \frac{a}{2-a} u\right) \Gamma\left(-\frac{1}{1-a}(t+1)-\frac{1}{1-a} u\right)}, \Gamma\left(-\frac{2}{2-a} u\right)
\end{aligned}
$$


with the following arguments of the generalized hypergeometric functions

$$
\begin{aligned}
\{\mathcal{A}\} & \equiv\left\{\alpha_{1}, \ldots, \alpha_{q}, \tilde{\alpha}_{1}, \ldots, \tilde{\alpha}_{(1-a) q}\right\} \\
\{\mathcal{B}\} & \equiv\left\{\beta_{1}, \ldots,\left.\beta_{i}\right|_{i \neq r+1}, \ldots, \beta_{q}, \tilde{\beta}_{1}, \ldots, \tilde{\beta}_{(1-a) q}\right\} \\
\alpha_{i} & =-\frac{2}{(2-a) q} u+\frac{r-1}{q}+\frac{i}{q}, \\
\tilde{\alpha}_{i} & =-\frac{2}{(2-a) q} u+\frac{r}{q}+\frac{m-2}{(1-a) q}+\frac{i}{(1-a) q}, \\
\beta_{i} & =1+\frac{r+1}{q}-\frac{i}{q} i \neq r+1, \\
\tilde{\beta}_{i} & =1-\frac{u}{(1-a) q}+\frac{r}{q}-\frac{i}{(1-a) q}, \\
\left\{\mathcal{A}^{\prime}\right\} & \equiv\left\{\alpha_{1}^{\prime}, \ldots, \alpha_{q}^{\prime}, \tilde{\alpha}_{1}^{\prime}, \ldots, \tilde{\alpha}_{(1-a) q}^{\prime}\right\} \\
\left\{\mathcal{B}^{\prime}\right\} & \equiv\left\{\beta_{1}^{\prime}, \ldots, \beta_{q}^{\prime}, \tilde{\beta}_{1}^{\prime}, \ldots,\left.\tilde{\beta}_{i}^{\prime}\right|_{i \neq t+1}, \ldots, \tilde{\beta}_{(1-a) q}^{\prime}\right\} \\
\alpha_{i}^{\prime} & =\frac{a}{2-a} \frac{u}{(1-a) q}+\frac{t+1}{(1-a) q}-\frac{1}{q}+\frac{i}{q}, \\
\tilde{\alpha}_{i}^{\prime} & =\frac{a}{2-a} \frac{u}{(1-a) q}+\frac{t+m-1}{(1-a) q}+\frac{i}{(1-a) q} \\
\beta_{i}^{\prime} & =1+\frac{t+1}{(1-a) q}+\frac{u}{(1-a) q}+\frac{1}{q}-\frac{i}{q}, \\
\tilde{\beta}_{i}^{\prime} & =1+\frac{t+1}{(1-a) q}-\frac{i}{(1-a) q} i \neq t+1 .
\end{aligned}
$$

Eq. (58) inserted into Eq. (48) gives the final answer for the collinear contribution. For $a=0$ or equivalently $p=q=1$, we reproduce the collinear part of (29), of course.

The poles in $u$ can be read off from (58), using the properties of the Gamma- and hypergeometric functions involved [31, 32]. The $\Gamma$-function $\Gamma(z)$ has simple poles at $z=-n, n$ integer, with residues $(-1)^{n} / n$ !, see Eq. (55), the hypergeometric ${ }_{p} F_{q}$ (57) has simple poles only in $\beta_{j}$ at $\beta_{j}=-n, n$ integer, with residues

$$
\frac{(-1)^{n}}{n !}{ }_{p} \tilde{F}_{q}\left(\left\{\alpha_{i}\right\} ;\left\{\beta_{1}, \ldots, \beta_{j-1},-n, \beta_{j+1}, \ldots, \beta_{q}\right\} ; z\right) \text {, }
$$

where ${ }_{p} \tilde{F}_{q}$ denotes the regularized hypergeometric function

$$
{ }_{p} \tilde{F}_{q}\left(\left\{\alpha_{1}, \ldots, \alpha_{p}\right\} ;\left\{\beta_{1}, \ldots, \beta_{q}\right\} ; z\right) \equiv \sum_{n=0}^{\infty} \frac{z^{n}}{n !} \frac{\prod_{i=1}^{p}\left(\alpha_{i}\right)_{n}}{\prod_{j=1}^{q} \Gamma\left(n+\beta_{j}\right)} .
$$


This means, that despite its appearance, (158) has poles in $u$ only in the $\Gamma$-functions, and

in the $\tilde{\beta}_{i}$ and $\beta_{i}^{\prime}$. Note that any pole in $u$ at $u=\rho$ is accompanied by a factor of $\nu^{\frac{2 \rho}{2-a}}$, resulting in general in a contribution of order $\mathcal{O}\left(\nu^{\frac{1}{2-a}}\left(\Lambda_{\mathrm{QCD}} / Q\right)\right)^{2 \rho}$.

\section{Acknowledgements}

We thank Mauro Anselmino, Einan Gardi, and George Sterman for helpful comments. L.M. thanks the CERN PH Department (Theory Unit) for hospitality during part of this work.

\section{References}

[1] E. Farhi, Phys. Rev. Lett. 39, 1587 (1977).

[2] H. Georgi and M. Machacek, Phys. Rev. Lett. 39, 1237 (1977);

G. Parisi, Phys. Lett. B 74, 65 (1978);

J. F. Donoghue, F. E. Low and S. Y. Pi, Phys. Rev. D 20, 2759 (1979);

G. C. Fox and S. Wolfram, Nucl. Phys. B 149, 413 (1979) [Erratum-ibid. B 157, $543(1979)]$;

R. K. Ellis, D. A. Ross and A. E. Terrano, Nucl. Phys. B 178 (1981) 421;

T. Chandramohan and L. Clavelli, Nucl. Phys. B 184, 365 (1981).

[3] S. Catani, G. Turnock and B. R. Webber, Phys. Lett. B 295, 269 (1992).

[4] G. Sterman, Nucl. Phys. B 281, 310 (1987).

[5] S. Catani, G. Turnock, B. R. Webber and L. Trentadue, Phys. Lett. B 263, 491 (1991).

[6] S. Catani, L. Trentadue, G. Turnock and B. R. Webber, Nucl. Phys. B 407, 3 (1993).

[7] Y. L. Dokshitzer, A. Lucenti, G. Marchesini and G. P. Salam, JHEP 9801, 011 (1998) hep-ph/9801324.

[8] M. Dasgupta and G. P. Salam, J. Phys. G 30, R143 (2004) hep-ph/0312283.

[9] L. Magnea, On power corrections to event shapes, hep-ph/0211013. 
[10] H. Contopanagos and G. Sterman, Nucl. Phys. B 419, 77 (1994) hep-ph/9310313;

B. R. Webber, Phys. Lett. B 339, 148 (1994) hep-ph/9408222;

A. V. Manohar and M. B. Wise, Phys. Lett. B 344, 407 (1995) hep-ph/9406392.

Y. L. Dokshitzer and B. R. Webber, Phys. Lett. B 352, 451 (1995) hep-ph/9504219|;

R. Akhoury and V. I. Zakharov, Nucl. Phys. B 465, 295 (1996) hep-ph/9507253;

M. Beneke, V. M. Braun and L. Magnea, Nucl. Phys. Proc. Suppl. 54A, 183 (1997) arXiv:hep-ph/9609266;

M. Beneke, V. M. Braun and L. Magnea, Nucl. Phys. B 497, 297 (1997) hep-ph/9701309.

[11] G. P. Korchemsky and G. Sterman, Nucl. Phys. B 437, 415 (1995) hep-ph/9411211;

G. P. Korchemsky and G. Sterman, in Moriond 1995: Hadronic:0383-392 hep-ph/9505391.

[12] Y. L. Dokshitzer, G. Marchesini and B. R. Webber, Nucl. Phys. B 469, 93 (1996) hep-ph/9512336;

Y. L. Dokshitzer, A. Lucenti, G. Marchesini and G. P. Salam, JHEP 9805, 003 (1998) arXiv:hep-ph/9802381;

M. Dasgupta, L. Magnea and G. Smye, JHEP 9911, 025 (1999) arXiv:hep-ph/9911316.

[13] E. Gardi, JHEP 0004, 030 (2000) hep-ph/0003179.

[14] P. A. Movilla Fernandez, S. Bethke, O. Biebel and S. Kluth, Eur. Phys. J. C 22, 1 (2001) hep-ex/0105059;

S. Bethke, J. Phys. G 26, R27 (2000) hep-ex/0004021;

S. J. Burby and C. J. Maxwell, Nucl. Phys. B 609, 193 (2001) hep-ph/0011203;

G. Dissertori, Measurements of alpha(s) from event shapes and the four-jet rate, hep-ex/0209070.

[15] G. P. Korchemsky, Shape functions and power corrections to the event shapes, in Minneapolis 1998, Continuous advances in QCD, 179 (1998) hep-ph/9806537;

G. P. Korchemsky and G. Sterman, Nucl. Phys. B 555, 335 (1999) hep-ph/9902341.

[16] G. P. Korchemsky, S. Tafat, JHEP 0010, (2000) 010 hep-ph/0007005;

A. V. Belitsky, G. P. Korchemsky, G. Sterman, Phys. Lett. B 515, (2001) 297 hep-ph/0106308.

[17] E. Gardi and J. Rathsman, Nucl. Phys. B 609, 123 (2001) hep-ph/0103217.

[18] E. Gardi and J. Rathsman, Nucl. Phys. B 638, 243 (2002) hep-ph/0201019.

[19] E. Gardi and L. Magnea, JHEP 0308, 030 (2003) hep-ph/0306094. 
[20] C. F. Berger, T. Kúcs and G. Sterman, Int. J. Mod. Phys. A 18, 4159 (2003) hep-ph/0212343;

C. F. Berger, T. Kúcs and G. Sterman, Phys. Rev. D 68, 014012 (2003) hep-ph/0303051.

[21] G. Sterman, Int. J. Mod. Phys. A 18, 4329 (2003) [Annales Henri Poincare 4, S259 (2003)] hep-ph/0301243.

[22] C. F. Berger, Soft Gluon Exponentiation and Resummation, Ph.D. Thesis, SUNY at Stony Brook, May 2003, UMI-30-98812 hep-ph/0305076.

[23] C. F. Berger and G. Sterman, JHEP 0309, 058 (2003) hep-ph/0307394;

C. F. Berger and G. Sterman, Power corrections to e+ $e$ - dijet event shapes, hep-ph/0310058.

[24] E. Gardi, Nucl. Phys. B 622, 365 (2002) hep-ph/0108222.

[25] E. Gardi and G. Grunberg, JHEP 9911, 016 (1999) hep-ph/9908458.

[26] M. Cacciari and E. Gardi, Nucl. Phys. B 664, 299 (2003) hep-ph/0301047;

E. Gardi, JHEP 0404, 049 (2004) hep-ph/0403249.

[27] E. Gardi, G. P. Korchemsky, D. A. Ross and S. Tafat, Nucl. Phys. B 636, 385 (2002) hep-ph/0203161;

E. Gardi and R. G. Roberts, Nucl. Phys. B 653, 227 (2003) hep-ph/0210429.

[28] G. P. Salam and D. Wicke, JHEP 0105, 061 (2001) hep-ph/0102343.

[29] S. Catani and M. H. Seymour, Phys. Lett. B 378, 287 (1996) hep-ph/9602277.

[30] P. Ball, M. Beneke and V. M. Braun, Nucl. Phys. B 452, 563 (1995) hep-ph/9502300.

[31] I. S. Gradshteyn and I. M. Ryzhik, Table of Integrals, Series, and Products, eds. A. Jeffrey and D. Zwillinger, 6th edition, Academic Press, London, 2000.

[32] Wolfram Research, Inc., http://functions. wolfram.com, 2004. 